\title{
Identification Strategy for Boundary Nodes of Reactive/Voltage Control Partition
}

\author{
Zhou Jing \\ Beijing DianyanHuayuan Electric Power Technology \\ Co., Ltd. \\ State Grid Electric Power Research Institute \\ Beijing, China \\ e-mail: zhoujing_0419@126.com

\section{Yan Hui} \\ China EPRI Science \&Technology Co., Ltd. \\ State Grid Electric Power Research Institute \\ Beijing, China \\ e-mail:pr_yanhui@sgri.sgcc.com.cn \\ Zhang Wenbin \\ Beijing DianyanHuayuan Electric Power Technology \\ Co., Ltd. \\ State Grid Electric Power Research Institute \\ Beijing, China \\ e-mail: keaiser@163.com
}

\author{
Li Hua \\ China EPRI Science \&Technology Co., Ltd. \\ State Grid Electric Power Research Institute \\ Beijing, China \\ e-mail: pr_lih@sgri.sgcc.com.cn
}

\section{Zi Zhenning}

China EPRI Science \&Technology Co., Ltd. State Grid Electric Power Research Institute Beijing, China e-mail:pr_zizn@sgri.sgcc.com.cn

\begin{abstract}
Partitioning the entire electrical network into several sub-regions is a good way to control voltage and reactive power. In this paper, an effective identification strategy is proposed to partition the whole network with typical boundary nodes. Firstly from the point of normal operation, a reactive sensitivity relationship matrix between reactive power sources is formulated to partition the reactive power source. These partitions ensure that there are the right amount of reactive power sources in each sub-region. Secondly, the sensitivity relationship matrix is calculated to figure out the comprehensive sensitivity between the reactive power source partition and load nodes. To solve the problem of the boundary nodes partition ambiguity, this paper presents an identification strategy based on perturbation increasing the load power. Through the analysis and calculation for IEEE39 node system, the simulation results achieve the apparent division to the boundary nodes. The partitions avoid the ambiguity effectively. The simulation results with IEEE39 node system demonstrate the effectiveness of this partition method.
\end{abstract}

Keywords-partitioning control of reactive power/voltage ; boundary nodes; identification strategies; electrical distance matrix; mapping partitioning algorithm

\section{INTRODUCTION}

Voltage hierarchical control scheme is widely used in power system operation. There are many countries in the world currently using this control scheme[1][2]. Reactive power and voltage distribution has the regional characteristic in large-scale power systems. In order to guarantee the control effect of reactive power and voltage, it is common to partition the entire network into several sub-region. It is necessary to have an accurate understanding of the physical power system during the partitioning the entire large complex networks which contain many reactive power sources. Thus, the partition results can be able to adapt to the actual situation of the power system. These partitions will strengthen internal electrical contact between nodes within a partition, reduce the degree of electrical coupling between partitions. In fact, there are always some nodes which will belong to several partition, these nodes are named as boundary node. The reasonable partition can avoid excessive problems of the ownership of the boundary nodes.

The typical partitioning methods are always based on the electrical distance calculated between the nodes[3-9]. According the electrical distance, the main consideration focused on two aspects, one scheme is first partitioning the reactive power nodes followed by load nodes[3] and the other scheme is first partitioning load nodes followed by partitioning the reactive power nodes[6]. For each node, mapping partitioning method[3], clustering algorithm[4][5] were commonly used to form the control partitions. Reference [6] adapted a short-circuit impedance ideas to get the electrical distance between the sub-region with many reactive source and a single load node. These references all used IEEE39 node system as the simulation case, but their partitioning results were slightly different. The results are displayed on a different partition on the typical processing boundary nodes. When the calculation of the sensitivity of some of the nodes, there are two or more partitions whose sensitivity are relatively close each other. These nodes are the boundary nodes. Therefore, it is 
necessary to adapt an identification strategy to give out the apparent membership of the boundary nodes.

In this paper, firstly from the point of normal operation, a reactive sensitivity relationship matrix between reactive power sources is formulated to partition the reactive power source. These partitions ensure that there are the right amount of reactive power sources in each sub-region. Secondly, the sensitivity relationship matrix is calculated to figure out the comprehensive sensitivity between the reactive power source partition and load nodes. To solve the problem of the boundary nodes partition ambiguity, this paper presents an identification strategy based on perturbation increasing the load power. Through the analysis and calculation for IEEE39 node system, the simulation results achieve the apparent division to the boundary nodes. The partitions avoid the ambiguity effectively. The simulation results with IEEE39 node system demonstrate the effectiveness of this partition method.

\section{REACTIVE POWER SOURCE SENSITIVITY MATRIX CALCUlation}

Reactive power and voltage control In the power system has the characteristic of spatially local distribution. In order to achieve effectively regulation of reactive power and voltage, there are the the need for decoupling control with a partition for the entire network. The key of decoupling is to seek a regional/nodal reactive/voltage change on the another node reactive/voltage variation sensitivity relationship. The reactive power voltage sensitivity of solving theoretical content is more classic. The basic idea is based on the Jacobian matrix of the power flow equations. The common solutions are Newton Raphson method and the PQ decomposition method.

For the study grid, using reactive correction equation of PQ decomposition method[8] can be expressed as

$$
\left[\begin{array}{c}
\Delta \boldsymbol{Q}_{G} \\
\Delta \boldsymbol{Q}_{L}
\end{array}\right]=\left[\begin{array}{ll}
\boldsymbol{B}_{G G} & \boldsymbol{B}_{G L} \\
\boldsymbol{B}_{L G} & \boldsymbol{B}_{L L}
\end{array}\right]\left[\begin{array}{l}
\Delta \boldsymbol{V}_{G} \\
\Delta \boldsymbol{V}_{L}
\end{array}\right]=\left[\boldsymbol{B}^{\prime \prime}\right] \bullet\left[\begin{array}{c}
\Delta \boldsymbol{V}_{G} \\
\Delta \boldsymbol{V}_{L}
\end{array}\right]
$$

In the formula, $\Delta \boldsymbol{Q}_{G} 、 \Delta \boldsymbol{Q}_{L}$ are the injection increment of reactive source and load node; $\Delta \boldsymbol{V}_{G} 、 \Delta \boldsymbol{V}_{L}$ are the node voltage increments of power source and the load; $\boldsymbol{B}_{G G} 、 \boldsymbol{B}_{G L} 、 \boldsymbol{B}_{L G}$ and $\boldsymbol{B}_{L L}$ is the coefficient matrix.

In the equation (1), B" should be a full-dimensional sensitivity matrix. It contains the PV node on the diagonal elements of the matrix to increase the number to ensure that this node voltage can maintain constant[4]. At this point matrix B "matrix structure is as follows.

$$
\left[\boldsymbol{B}^{\prime \prime}\right]=\left[\begin{array}{cccccc}
B_{11} & \Lambda & B_{1, N G} & B_{1, N G+1} & \Lambda & B_{1 n} \\
B_{21} & \Lambda & B_{2, N G} & B_{2, N G+1} & \Lambda & B_{2 n} \\
B_{31} & \Lambda & B_{3, N G} & B_{3, N G+1} & \Lambda & B_{3 n} \\
\Lambda & \Lambda & \Lambda & \Lambda & \Lambda & \Lambda \\
B_{n 1} & \Lambda & B_{n, N G} & B_{n, N G+1} & \Lambda & B_{n n}
\end{array}\right]
$$

In the formula, $\mathrm{n}$ is the number of independent nodes except balance node; NG is the number of reactive power source which are PV node; the serial number of the balance node is $\mathrm{n}+1$.

In the matrix B", these NG front column of the matrix are set larger number to ensure that when calculating the sensitivity of such nodes can guarantee constant voltage; rest of the columns can be calculated by normal network, such as the formation of node admittance matrix .

To determine the sensitivity of the relationship between reactive power sources can make $\Delta \boldsymbol{Q}_{L}=0$, the equation can be simplified as

$$
\Delta \boldsymbol{Q}_{G}=\left[\boldsymbol{B}_{G G}-\boldsymbol{B}_{G L} \boldsymbol{B}_{L L}^{-1} \boldsymbol{B}_{L L}\right] \Delta \boldsymbol{V}_{G}=\boldsymbol{S}_{G G} \Delta \boldsymbol{V}_{G}
$$

In the formula, the coefficient matrix $\boldsymbol{S}_{G G}$ is the sensitivity matrix between reactive power injection and voltage, it is a symmetric matrix. According to this sensitivity matrix, reactive power source can be partitioned.

\section{COMPREHENSIVE SENSITIVITY FOR PARTITIONING LOAD NODES}

Generally there are several reactive power sources in every partition. A load node belongs to a certain partition, that meas the integrated control of reactive power source in this partition to the load node is stronger than the other partitions. There is fewer electrical distance than the other partitions. This paper presents an improved methods to calculate the comprehensive sensitivity between the reactive power source and a single node.

\section{A. Comprehensive Sensitivity Calculation Process}

Suppose the number of reactive power sources is NG, partitions has been formed in accordance with Section II. There are $\mathrm{NG}_{\mathrm{j}}$ reactive power sources in the $\mathrm{j}^{\text {th }}$ partition, the number of reactive power partition is $\mathrm{m}$. The number of load node is NL. The comprehensive sensitivity between $\mathrm{NG}_{\mathrm{j}}$ and load node $\mathrm{i}$ is calculated as follows.

- Input the data, including the electricity network data, reactive sources data, load data; then uses the PQ decomposition method program to calculate the load flow results.

- Perturbs the load power $\Delta \tilde{S}_{i}$ of load node $i \in L$, keep the power of other load nodes constant. All the nodes with the zero power in the network are considered as the common load nodes.

- For the reactive source node in $\mathrm{NG}_{\mathrm{j}}$, set it as a PV node if it participates in primary voltage control, otherwise set to PQ node. Usually only one node is set as a balanced node, its voltage and angle remains constant.

- Calculate the load flow considering $\Delta \tilde{S}_{i}$, the comprehensive sensitivity is calculated as follows.

$$
\alpha_{N G_{j}}^{i}=\frac{\sum_{k \in N G_{j}} \Delta Q_{k}}{\Delta Q_{i}}
$$


- Restore the power of load node; return to the second step to calculate the next node sensitivity.

- According to the comprehensive sensitivity, a comprehensive sensitivity matrix between the reactive power source partition and load node is established as follows.

$$
\boldsymbol{\alpha}_{G}^{L}=\left[\begin{array}{cccc}
\alpha_{N G_{1}}^{1} & \alpha_{N G_{2}}^{1} & \Lambda & \alpha_{N G}^{1} \\
\alpha_{N G_{1}}^{2} & \alpha_{N G_{2}}^{2} & \Lambda & \alpha_{N G}^{2} \\
\Lambda & \Lambda & \Lambda & \Lambda \\
\alpha_{N G_{1}}^{L} & \alpha_{N G_{2}}^{L} & \Lambda & \alpha_{N G}^{L}
\end{array}\right]
$$

\section{B. Mapping Partitioning Load Nodes}

With the comprehensive sensitivity matrix in formula (5), the electrical distance between load node $\mathrm{i}$ and partition $\mathrm{NG}_{\mathrm{j}}$ is shown as follows.

$$
d_{i j}=-\lg \left|\alpha_{N G_{j}}^{i}\right|
$$

In formulation (5), negative sign converts the logarithmic value to be positive. The smaller is $d_{i j}$, the closer indicates the load node $\mathrm{i}$ and partition $\mathrm{j}$. It also means that $j^{\text {th }}$ partition has the stronger comprehensive control capability of the load node i than other partitions. So the electrical distance $\mathrm{d}_{\mathrm{ij}}$ can accurately measure the electrical closeness between a reactive power source partition and a single load node.

Thus the electrical distance matrix $\mathrm{D}$ can be formed between the reactive power source partition and load node on the basis of formula (6), which is as follows:

$$
\boldsymbol{D}=\left[\begin{array}{cccc}
d_{11} & d_{12} & \Lambda & d_{1, m} \\
d_{21} & d_{22} & \Lambda & d_{2, m} \\
\Lambda & \Lambda & \Lambda & \Lambda \\
d_{N L, 1} & d_{N L, 2} & \Lambda & d_{N L, m}
\end{array}\right]
$$

In formulation (7), the row number is NL, the column number equals partition number $\mathrm{m}$. In matrix $\mathrm{D}$, the $\mathrm{k}^{\text {th }}$ row $k \in m$ indicates the electrical distance between the load node $\mathrm{k}$ and all the partitions. The map partitioning algorithm uses the minimum electrical distance principle to partition the load node into the nearest reactive source partition.

\section{IDENTIFICATION STRATEGIES OF BOUNDARY NODES}

According to the definition of the sensitivity, the comprehensive sensitivity is calculated with the load flow in a cross-section of power system operation. Therefore, the operating state of the power system has great influence on the partition results. Different sensitivity calculation conditions also led to the sensitivity of the results have some differences on the partition results.

For example, in the typical case IEEE39 node system, [3], [4], [8], [9] are using the idea of electrical sensitivity, but there are some different partition results. This difference focus on the boundary nodes 17,26 and 27 . The reactive power source partition results are consistent in all of these references. Obviously, different partitions will have a significant impact on the subsequent reactive power and voltage control strategies. Sometimes this impact maybe appear greatly .

When calculating sensitivity of the nodes, there are two or more partitions whose sensitivity are relatively close each other. These nodes are the boundary nodes. To be able to partition reasonably, the boundary nodes must be given special treatment. If according to the maximum partition ownership directly, there is a certain point or a little far-fetched to partition these boundary nodes.

For the different partition in [3][4][8][9], an identification strategy is used to partition the boundary nodes with the perturbed incremental load to calculate multiple sensitivity. On the basis of the load flow, gradually increasing the reactive load power of a boundary node perturbation. Perturbation values can take from 0.01 to 0.5 (p.u.) or more.

For the actual system operation, the load increment often vary greatly, not necessarily equal to the calculated sensitivity when sensitivity calculation. So constantly increasing load changes can get the control law of different reactive source partition for the boundary nodes. The kind of change trend can identify the membership of reactive power partition. This avoids the partition ambiguity only from the sensitivity of the cross-section data. These serial increment can be achieved effectively to identify boundary nodes partition.

\section{CASE STUDY}

To verify the feasibility of the proposed identification strategy, the typical IEEE39 node system is used as the simulation case partition reactive power sources and load nodes. When partitioning the reactive power sources, the 10 reactive sources (node 30- node 39) are divided into six partitions. On the basis of these partitions, the rest of the load nodes are partitioned in turn with the comprehensive sensitivity matrix. The partition result is shown in Table 1.

As can be seen from Table 1, the six reactive power sources and majority of the load nodes partitions are the same with all these methods. But there are three load nodes partitioning results, 17,26 and 27 , have some differences. These three nodes are the boundary nodes, different operation may have different effect on them, even different perturbation size will have an apparently impact on the partition results while seeking the comprehensive sensitivity calculation.

In the actual operation of power system, the amplitude of the load changes may be large or small. During the process of calculating the sensitivity, the size of the perturbation values also affect the partitioning results, leading to the fluctuations of the comprehensive sensitivity boundary. An identification strategy is to increase gradually the load power of boundary nodes. To a certain extent, this strategy can simulate the actual load fluctuations in the power system operation. And this growth can reflect the different variation of reactive power sources partition control of the boundary nodes .

From Fig .1 to Fig .3, the partition results from the value of reactive power perturbation from 0.05 to 0.5 (p.u.), which give a partition changes about the boundary nodes 
17, 26 and 27. In Fig .1 to Fig .3, ordinate axis indicates electrical distance, the horizontal axis represents the reactive source partition.

TABLEI. DIFFERENT PARTITION OF IEEE 39-BUS SYSTEM

\begin{tabular}{|c|c|c|c|c|}
\hline $\begin{array}{l}\text { Partitio } \\
\text { n No. }\end{array}$ & $\begin{array}{l}\text { Results of } \\
\text { This paper }\end{array}$ & $\begin{array}{c}\text { Results of } \\
{[3]}\end{array}$ & $\begin{array}{c}\text { Results of } \\
{[4]}\end{array}$ & $\begin{array}{c}\text { Results of } \\
{[8]}\end{array}$ \\
\hline 1 & $\begin{array}{c}2,3,18,25 \\
26,27 \\
30 *, 37 *\end{array}$ & $\begin{array}{c}2,3,18,25 \\
30^{*}, 37^{*}\end{array}$ & $\begin{array}{l}2,3,17,18, \\
25,26,27 \\
30^{*}, 37^{*}\end{array}$ & $\begin{array}{c}2,3,17,18, \\
25,26,27 \\
30^{*}, 37^{*}\end{array}$ \\
\hline 2 & $1,9,39 *$ & $1,9,39 *$ & $1,9,39^{*}$ & $1,9,39 *$ \\
\hline 3 & $\begin{array}{c}4,5,6,7,8, \\
10,11,12,1 \\
3,14, \\
31 *, 32 *\end{array}$ & $\begin{array}{c}4,5,6,7,8 \\
10,11,12,1 \\
3,14, \\
31^{*}, 32 *\end{array}$ & $\begin{array}{c}4,5,6,7,8 \\
10,11,12,1 \\
3,14 \\
31^{*}, 32^{*}\end{array}$ & $\begin{array}{c}4,5,6,7,8, \\
10,11,12,1 \\
3,14, \\
31^{*}, 32^{*}\end{array}$ \\
\hline 4 & $\begin{array}{c}19,20,33^{*}, \\
34^{*}\end{array}$ & $\begin{array}{c}19,20,33^{*}, \\
34^{*}\end{array}$ & $\begin{array}{c}19,20,33^{*}, \\
34^{*}\end{array}$ & $\begin{array}{c}19,20,33^{*}, \\
34 *\end{array}$ \\
\hline 5 & $\begin{array}{c}15,16,17 \\
21,22,23, \\
24, \\
35^{*}, 36^{*}\end{array}$ & $\begin{array}{c}15,16,17 \\
21,22,23, \\
24, \\
35^{*}, 36^{*}\end{array}$ & $\begin{array}{c}15,16,21 \\
22,23,24 \\
35^{*}, 36^{*}\end{array}$ & $\begin{array}{c}15,16,21 \\
22,23,24 \\
35^{*}, 36^{*}\end{array}$ \\
\hline 6 & $28,29,38 *$ & $\underline{26}, \frac{27}{9,38 *}, 28,2$ & $28,29,38 *$ & $28,29,38 *$ \\
\hline
\end{tabular}

Note: The underlined node represents a boundary node, the nodes with * represent reactive source node.

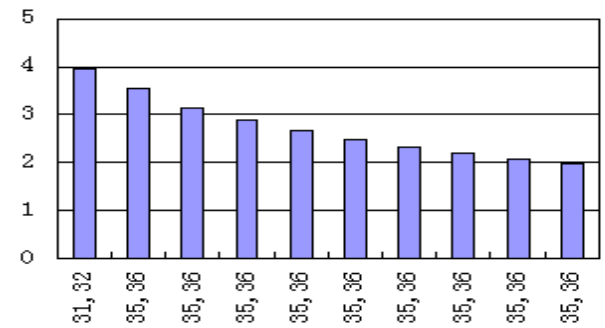

Figure 1. Relationship of electrical distance and partition of node 17

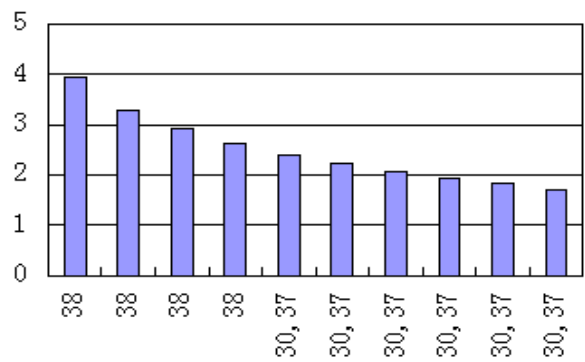

Figure 2. Relationship of electrical distance and partition of node 26

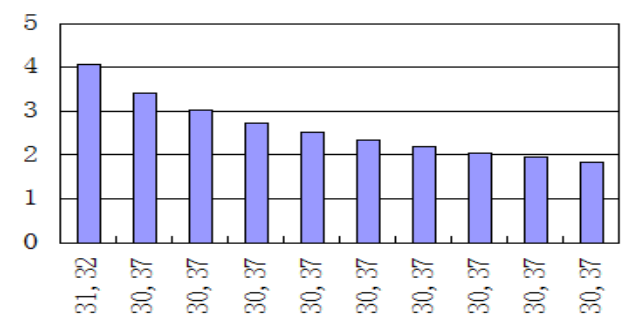

Figure 3. Relationship of electrical distance and partition of node 27
Variation can be seen from the above three figures. the selection of perturbation calculations have a great impact on sensitivity. When choosing a smaller perturbation 0.05 , partitions and larger perturbation boundary nodes are not entirely consistent. When selected perturbation above 0.25 , the partition under the three boundary nodes stabilized and clear, the partition does not change. Therefore, the identification strategy based on increasing perturbation can avoid the personal experience uncertainty of perturbation selection. The results of multiple partitions under the boundary nodes can be clear, accurate and easily achieve the reasonable partition.

\section{CONCLUSIONS}

Reactive sensitivity relationship matrix is formulated to partition the reactive power sources. The sensitivity relationship matrix is calculated to partition and load nodes. To solve the problem of the boundary nodes partition ambiguity, an effective identification strategy is proposed to partition the whole network with typical boundary nodes. The simulation results with IEEE39 node system demonstrate the effectiveness of this identification strategy.

\section{REFERENCES}

[1] Lagonotte P, Sabonnadiere J C, Leost J Y, et al. "Structural analysis of the electrical system application to secondary voltage control in France", IEEE Trans on Power Systems, vol.4,1989, pp:479-486.

[2] Sancha J L, Fernandez J L, Cortes A, et al. "Secondary voltage control analysis solutions and simulation results for the Spanish transmission system", IEEE Trans on Power Systems, vol.11,1996, pp:630-638.

[3] ZHAO Jin quan, LIU Fu cheng, DENG Yong, et al. "Network partitioning for reactive power/voltage control based on a mapping division algorithm", Automation of Electric Power Systems, vol.34, 2010,pp:36-39.

[4] GUO Qinglai, SUN Hongbin, ZHANG Boming, et al. "Power network partitioning based on clustering analysis in Mvar control spacev, Automation of Electric Power Systems, vol.29, 2005 , pp:36-40.

[5] HU Zechun, WANG Xifan, WANG Xiuli, et al. "Two-layered network partitioning approach for optimal reactive power dispatching", Power System Technology, vol.29, 2005,pp:37-41.

[6] LIU Jun, BAI Qiang, XIE Xiao mei, et al. "Study of secondary voltage control using fuzzy clustering electric distance method", Power System Technology, vol.30, 2006, pp:250-254

[7] WU Jixiang, TU Guangyu, LUO Yi. "A real-time region identification based on fuzzy pattern recognition for distributed voltage control", Automation of Electric Power Systems, vol.30, 2006, pp:36-41.

[8] YAN Wei, WANG Fang, TANG Wen-zuo, et al. "Network partitioning for reactive power/voltage control based on power sources clustering and short-circuit impedance distance", Power System Protection and Control, 2013, 41(7):109-115.

[9] Yang Xiuyuan, Dong Zheng, Tang Bao, et al. "Power Network Partitioning Based on Fuzzy Clustering Analysis", Proceedings of the CSEE, vol.26,2006,pp:6-10.

[10] .Quintana V. H, Muller N. "Partitioning of Power Networks and Applications to Security Control", IEE Proceedings, 1991,138(6): $535-545$ 